\title{
Two-Dimensional Stress Analysis of Thick Hollow Functionally Graded Sphere Under Non-Axisymmetric Mechanical Loading
}

\author{
Sandeep Kumar Paul \\ Department of Mathematics, School of Technology, \\ Pandit Deendayal Energy University, Gandhinagar, Gujarat, 382426, India. \\ E-mail: sandeep.kphd17@sot.pdpu.ac.in \\ Manoj Sahni \\ Department of Mathematics, School of Technology, \\ Pandit Deendayal Energy University, Gandhinagar, Gujarat, 382426, India. \\ Corresponding author: manoj.sahni@sot.pdpu.ac.in
}

(Received on February 27, 2021; Accepted on May 1, 2021)

\begin{abstract}
In this paper, a functionally graded thick hollow sphere is considered for the analysis of two-dimensional steady state mechanical stress in the radial and circumferential directions under mechanical loading. Modulus of elasticity is varying with continuous nonlinear variation along the radial direction and Poisson's ratio is kept as constant. The Legendre series and Euler differential equation are used to solve Navier equations. Geometry of the sphere is assumed in spherical coordinate system. Applying mechanical boundary conditions at inner and outer radii, we have carried out the analytical solutions for stresses, strains and displacements. In the numerical example, only internal pressure is varying along circumferential direction and external pressure is kept as zero. Displacements and mechanical stresses are presented graphically and the results are discussed numerically.
\end{abstract}

Keywords- Sphere, Young's modulus, Stresses, Pressure, Functionally graded material.

\section{Introduction}

In the beginning, Functionally Graded Materials (FGMs) were used to design heat resisting material in the aerospace industry. But by the passage of time, the concept of FGM is applied in many structural problems by engineers and researchers. Usually, these materials are used to design several structural components to optimize the response of component under thermal and mechanical loading. Basically, functionally graded materials are the new kind of nonhomogeneous engineering materials in which composition and structure change continuously over the volume (Xu et al., 2019). By handling the change in the mechanical and thermal properties of the material in different dimensions, engineers can design multipurpose device (Aragh and Hedayati, 2012). Prediction is made for the partial behaviour of stresses of thick-walled functionally graded cylinder subjected to uniform inner and outer pressure along thickness direction by Nejad and Fatehi (2015). Using Frobenius series method, a cylindrical pressure vessel made of functionally graded material is investigated as elasticity problem by Gharibi et al. (2017). Sahni and Sharma (2017) have carried out the results of elastic-plastic stresses in a rotating solid disk with varying density in thickness direction followed by an exponential law.

In recent years, many researchers have worked for the analysis of thermo-mechanical stresses for one-dimensional functionally graded material. But there are only few researchers that are working on $2 \mathrm{D}$ and 3D thermo-mechanical problems under steady and unsteady state conditions. A long functionally graded hollow cylinder is analysed under internal pressure and uniform heat generation 
by Evci and Gülgeç (2018). A functionally graded hollow cylinder is investigated analytically for thermal and mechanical stresses under non-axisymmetric steady state loads by Jabbari et al. (2003). An infinite length cylinder made of functionally graded material is analysed for two-dimensional thermos elastic problem under unstable condition using Green's function approach following laminate theory (Kim and Noda, 2002). Wang and Noda (2001) adopted integral transform method to carry out the results on failure status of a damaged functionally graded actuator on a surface under temperature load.

A two-dimensional axisymmetric problem is solved to explore the response of wave propagation using finite element method for a thick hollow cylinder formed of functionally graded material with finite length under effective internal pressure (Asgari et al., 2009). Loghman et al. (2017) have solved a no symmetric thermo-elastic problem analytically for FGM cylinder under mechanical and thermal loadings subjected to uniform magnetic field. A thick-walled pressure vessel of spherical shape having inner FGM coating is investigated for elastic and plastic stresses under the external and internal pressures (Seyyed Nosrati et al., 2019). Using linear theory of elasticity, a threedimensional homogeneous isotropic rotating cylindrical panel made of functionally grade material is examined for wave propagation by Selvamani and Ponnusamy (2011). Using Finite element method, Mehta and Sahni (2020) have done stress analysis for functionally graded rotating disc under the influence of internal pressure, centrifugal body forces and thermal loading in radial direction. Natural frequencies of free vibrations are studied for isotropic, spherically symmetric, thermos-elastic and FG (Functionally Graded) sphere using Laplace Transform without implementing its inversion (Sharma and Mishra, 2017). Theory of elasticity in uncoupled form is utilized to inspect the influence of heat source on temperature stresses in functionally graded solid sphere as a fusion of ceramic $\mathrm{ZrO}_{2}$ and alloy Ti-6A1-4V (Pawar et al., 2017).

Paul and Sahni (2019) used Power series method to derive two-dimensional stresses of an axisymmetric cylindrical pressure vessel made of FGM. Spherical shell, cylindrical shell and plates made of functionally graded material are considered for the thermal stress analysis under different thermal loadings using finite element method by Reddy and Chin (1998). Stress analysis of a rotating disc along its thickness direction is investigated by Singh and Sahni (2016). Mahbadi (2017) has applied an approximation method to carry out the results of stress potency factor for functionally graded rotating solid cylinder having fracture in radial direction under plain stress and plain strain conditions subjected to thermal and mechanical loadings.

In this work, Young's modulus is varying with nonlinear variation in radial direction and Poisson's ratio is fixed as constant. Displacements are calculated in radial and tangential directions. Equilibrium equations are transformed into second order partial differential equations in displacement components and further solved by Legendre series. Employing Legendre series, we convert partial differential equation into homogeneous Euler differential equations. Applying harmonic boundary conditions on displacements and mechanical stresses we are able to find displacements, strains and stresses. Boundary conditions are changing along circumferential direction for this problem.

\section{Mathematical Formulation of Stress Analysis}

Assume a functionally graded thick hollow sphere with inner radius ' $r_{1}{ }^{\prime}$ and outer radius ' $r_{2}$ '. Spherical coordinate axes $(r, \theta, \phi)$ is considered for FGM sphere that represents radial distance, polar angle and azimuthal angle respectively and internal pressure $p_{1}(\theta)$, external pressure $p_{2}(\theta)$ are varying along tangential direction as shown in the Figure 1. 


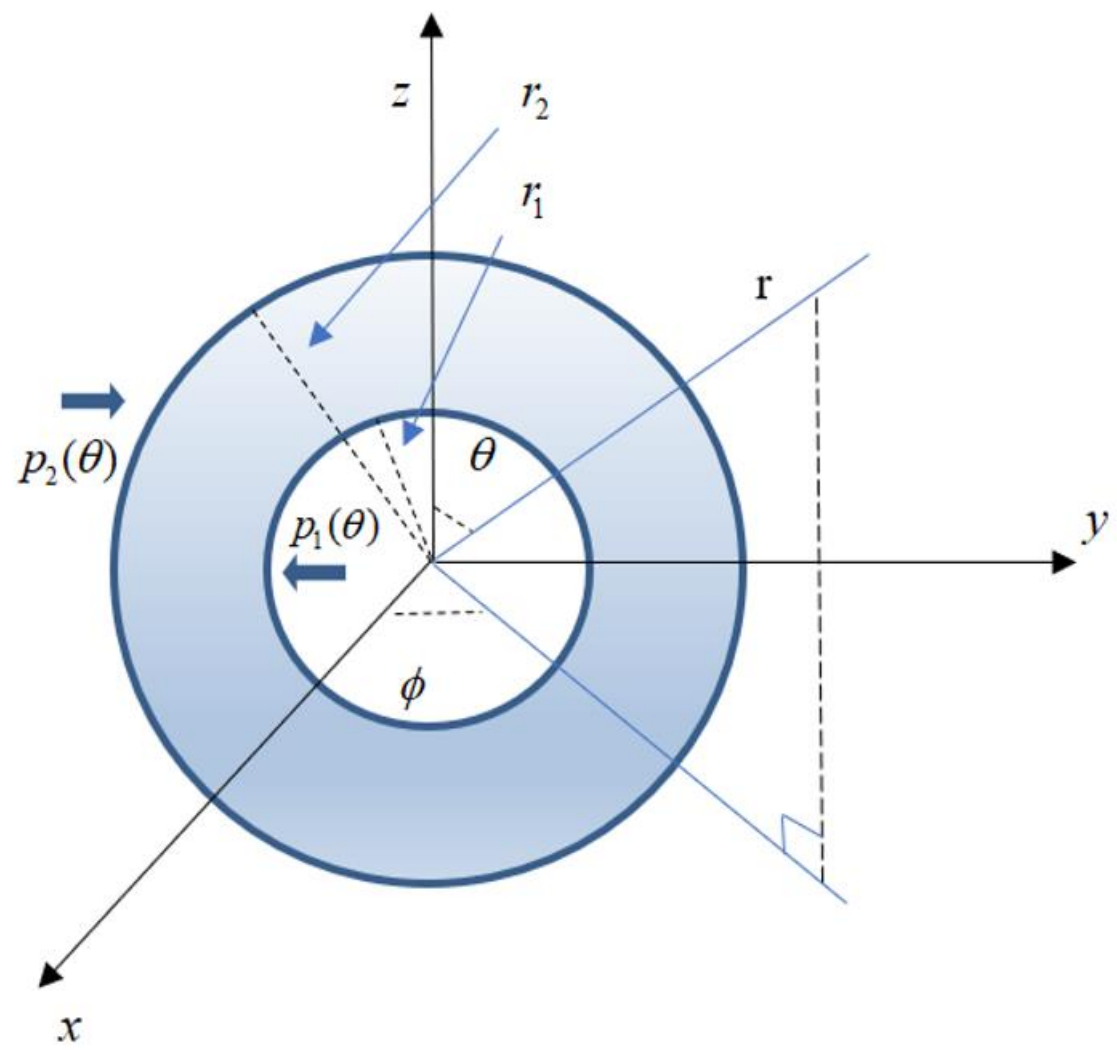

Figure 1. FGM Thick sphere with spherical coordinates.

Stresses, strains and displacements are varying along ' $r$ ' and ' $\theta$ ' dimensions. Modulus of elasticity has smooth and continuous variation along the radial direction and follows the nonlinear for

$Y(r)=Y_{0} r_{2} \beta \log (r)$

where $\beta \neq 0,-6 \leq \beta \leq 10$ and $Y_{0}$ refers the constant value of Young's modulus and ' $\beta$ ' is material parameter.

Hooke's law for isotropic material can be written as (Poultangari et al., 2008).

$$
\begin{aligned}
& \sigma_{r r}=(\xi+2 \eta) \varepsilon_{r r}+\xi \varepsilon_{\theta \theta}+\xi \varepsilon_{\phi \phi}, \sigma_{\theta \theta}=\xi \varepsilon_{r r}+(\xi+2 \eta) \varepsilon_{\theta \theta}+\xi \varepsilon_{\phi \phi} . \\
& \sigma_{\phi \phi}=\xi \varepsilon_{r r}+\xi \varepsilon_{\theta \theta}+(\xi+2 \eta) \varepsilon_{\phi \phi}, \sigma_{r \theta}=2 \eta \varepsilon_{r \theta}, \sigma_{\theta \phi}=\sigma_{r \phi}=0
\end{aligned}
$$

where $\sigma_{m n}$ and $\varepsilon_{m n}$ represent stress and strain tensors for $m, n=r, \theta, \phi$. Lame's constants ' $\xi$ ' and ' $\eta$ ' involving Young's modulus ' $Y(r)^{\prime}$ and Poisson's ratio ' $v$ ' are written as (Poultangari et al., 2008).

$\xi=\frac{v Y(r)}{(1+v)(1-2 v)}, \eta=\frac{Y(r)}{2(1+v)}$ 
Strain-displacement equations are assumed in the radial and circumferential directions as (Mahbadi, 2017).

$\varepsilon_{r r}=\frac{\partial u}{\partial r}, \varepsilon_{\theta \theta}=\frac{u}{r}+\frac{1}{r} \frac{\partial w}{\partial \theta}, \varepsilon_{\phi \phi}=\frac{u}{r}+\frac{w}{r} \cot \theta, \varepsilon_{r \theta}=\frac{1}{2}\left(\frac{1}{r} \frac{\partial u}{\partial \theta}+\frac{\partial w}{\partial r}-\frac{w}{r}\right)$

where ' $u$ ' and ' $w$ 'are components of radial and tangential displacements respectively.

Equilibrium stress equations, ignoring inertia and body forces in ' $r$ ' and ' $\theta$ ' dimensions are (Poultangari et al., 2008).

$\frac{\partial \sigma_{r r}}{\partial r}+\frac{1}{r}\left(\frac{\partial \sigma_{r \theta}}{\partial \theta}+2 \sigma_{r r}\right)-\frac{1}{r}\left(\sigma_{\theta \theta}+\sigma_{\phi \phi}-\sigma_{r \theta} \cot \theta\right)=0$

$\frac{\partial \sigma_{r \theta}}{\partial r}+\frac{1}{r}\left(\frac{\partial \sigma_{\theta \theta}}{\partial \theta}+3 \sigma_{r \theta}\right)+\frac{1}{r}\left(\sigma_{\theta \theta}-\sigma_{\phi \phi}\right) \cot \theta=0$

Inserting $\psi=\cos \theta$ and using equations (1) -(6), the displacement equations are as

$$
\begin{aligned}
& \frac{\partial^{2} u}{\partial r^{2}}+\left(\beta \log r_{2}+2\right) \frac{1}{r} \frac{\partial u}{\partial r}+\frac{2}{r^{2}}\left(\frac{v}{1-v} \beta \log r_{2}-1\right)+\frac{1}{2 r} \frac{1}{(1-v)} \frac{\partial}{\partial r}\left(w \cot \theta-\frac{\partial w}{\partial \psi} \sin \theta\right)+ \\
& \frac{1}{2 r^{2}} \frac{1-2 v}{1-v}\left(\left(1-\psi^{2}\right) \frac{\partial^{2} u}{\partial \psi^{2}}-2 \psi \frac{\partial u}{\partial \psi}\right)+\frac{1}{r^{2}}\left(\frac{v}{1-v} \beta \log r_{2}-\frac{3-4 v}{2-2 v}\right)\left(w \cot \theta-\frac{\partial w}{\partial \psi} \sin \theta\right)=0 \\
& \frac{\partial^{2} w}{\partial r^{2}}+\left(\beta \log r_{2}+2\right) \frac{1}{r} \frac{\partial w}{\partial r}-\frac{1}{r^{2}}\left(\beta \log r_{2}+\frac{1}{1-\psi^{2}} \frac{2-2 v}{1-2 v}\right) w-\frac{\sqrt{1-\psi^{2}}}{r(1-2 v)} \frac{\partial^{2} u}{\partial r \partial \psi} \\
& +\frac{2}{r^{2}} \frac{1-v}{(1-2 v)}\left(\left(1-\psi^{2}\right) \frac{\partial^{2} w}{\partial \psi^{2}}-2 \psi \frac{\partial w}{\partial \psi}\right)-\frac{\sqrt{1-\psi^{2}}}{r^{2}}\left(\beta \log r_{2}+\frac{4-4 v}{1-2 v}\right) \frac{\partial u}{\partial \psi}=0
\end{aligned}
$$

The equations (7) and (8) represent Navier equations in the form of second order partial differential equations. Radial and tangential displacements are stated in the form of Legendre series as

$u(r, \psi)=\sum_{k=0}^{\infty} U_{k}(r) P_{k}(\psi), w(r, \psi)=\sum_{k=0}^{\infty} W_{k}(r) P^{\prime}{ }_{k}(\psi) \sqrt{1-\psi^{2}}$

where $U_{k}(r)$ and $W_{k}(r)$ are functions of $r$. Placing equation (9) into (7) and (8), and using recurrence relations of Legendre polynomial, we get

$$
\begin{aligned}
& \frac{d^{2} U_{k}(r)}{d r^{2}}+\left(\beta \log r_{2}+2\right) \frac{1}{r} \frac{d U_{k}(r)}{d r}+\frac{1}{r^{2}}\left(\frac{2 v}{1-v} \beta \log r_{2}-k(k+1) \frac{1-2 v}{2-2 v}-2\right) U_{k}(r) \\
& +\frac{k(k+1)}{2 r(1-v)} \frac{d W_{k}(r)}{d r}+\frac{k(k+1)}{r^{2}}\left(\frac{v}{1-v} \beta \log r_{2}-\frac{3-4 v}{2-2 v}\right) W_{k}(r)=0 \\
& \frac{d^{2} W_{k}(r)}{d r^{2}}+\left(\beta \log r_{2}+2\right) \frac{1}{r} \frac{d W_{k}(r)}{d r}-\frac{1}{r(1-2 v)} \frac{d U_{k}(r)}{d r}-\frac{1}{r^{2}}\left(\beta \log r_{2}+k(k+1) \frac{2-2 v}{1-2 v}\right) W_{k}(r)- \\
& \frac{1}{r^{2}}\left(\beta \log r_{2}+\frac{4-4 v}{1-2 v}\right) U_{k}(r)=0
\end{aligned}
$$


Equations (10) and (11) are representing homogeneous Euler ordinary differential equations whose general solution can be found by the method of substitution. Thus, the general solutions for these equations can be found by substituting.

$U_{k}(r)=R r^{\rho}, W_{k}(r)=S r^{\rho}$

where ' $\rho$ ' can be found using the equation (15) and $R$, Sare unknown constants.

Using equation (12) into the equations (10) and (11), we get

$$
\begin{aligned}
& \left(\rho^{2}+\left(\beta \log r_{2}+1\right) \rho+\frac{2 v \beta \log r_{2}}{1-v}-k(k+1) \frac{1-2 v}{2-2 v}-2\right) R+ \\
& \frac{k(k+1)}{2-2 v}\left(\rho+2 v \beta \log r_{2}-3+4 v\right) S=0 \\
& -\frac{1}{1-2 v}\left(\rho+(1-2 v) \beta \log r_{2}+4-4 v\right) R+\left(\begin{array}{c}
\rho^{2}+\left(\beta \log r_{2}+1\right) \rho \\
-\beta \log r_{2} \\
-k(k+1) \frac{2-2 v}{1-2 v}
\end{array}\right) S=0
\end{aligned}
$$

Equations (13) and (14) give homogeneous system of linear equations in $R$ and $S$.

Non-trivial solution from equations (13) and (14) can be obtained as

$$
\begin{aligned}
& \left(\rho^{2}+\left(\beta \log r_{2}+1\right) \rho+\frac{2 v \beta \log r_{2}}{1-v}-k(k+1) \frac{1-2 v}{2-2 v}-2\right) \\
& \times\left(\rho^{2}+\left(\beta \log r_{2}+1\right) \rho-\beta \log r_{2}-k(k+1) \frac{2-2 v}{1-2 v}\right)
\end{aligned}
$$

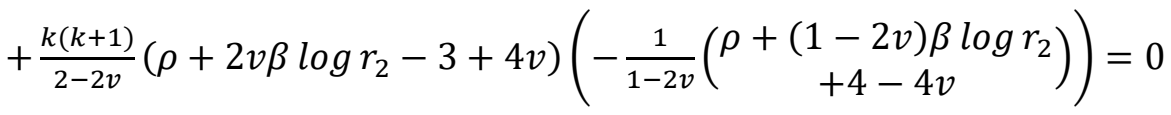

Solving equation (15) for $\rho$, we get four eigen values $\rho_{k i}(i=1,2,3,4)$ for different $k$, and using these values, general solutions for equation (12) are obtained as

$U_{k}(r)=\sum_{i=1}^{4} R_{k i} r^{\rho_{k i}}, W_{k}(r)=\sum_{i=1}^{4} D_{k i} R_{k i} r^{\rho_{k i}}$

where $S_{k i}=D_{k i} R_{k i}$ and $D_{k i}=\frac{\rho_{k i}^{2}+\left(\beta \log r_{2}+1\right) \rho_{k i}+\frac{2 v \beta \log r_{2}}{1-v}-k(k+1) \frac{1-2 v}{2-2 v}-2}{-\frac{k(k+1)}{2-2 v}\left(\rho_{k i}+2 v \beta \log r_{2}-3+4 v\right)}$.

If we consider $k=0$, then $P^{\prime}{ }_{k}(\psi)$ becomes zero. Thus, equations (10) and (11) are converted into one differential equation as

$$
\frac{d^{2} U_{0}(r)}{d r^{2}}+\left(\beta \log r_{2}+2\right) \frac{1}{r} \frac{d U_{0}(r)}{d r}+\frac{1}{r^{2}}\left(\frac{2 v}{1-v} \beta \log r_{2}-2\right) U_{0}(r)=0
$$


Solving above homogeneous ordinary differential equation, we get the general solution for $U_{0}(r)$

$U_{0}(r)=\sum_{i=1}^{2} R_{0 i} r^{\rho_{0 i}}$

where $\rho_{01}, \rho_{02}=\frac{-\left(\beta \log r_{2}+1\right) \pm\left(\left(\beta \log r_{2}+1\right)^{2}-8\left(\frac{v \beta \log r}{1-v}-1\right)\right)^{\frac{1}{2}}}{2}$

Thus, using the equations (16), (17) and (18), complete solutions of the displacements along radial and tangential directions for all inputs of $k$ are given as

$$
\begin{aligned}
& u(r, \psi)=\sum_{i=1}^{2} R_{0 i} r^{\rho_{0 i}}+\sum_{k=1}^{\infty}\left[\sum_{i=1}^{4} R_{k i} r^{\rho_{k i}}\right] P_{k}(\psi), \\
& w(r, \psi)=\sum_{k=1}^{\infty}\left[\sum_{i=1}^{4} D_{k i} R_{k i} r^{\rho_{k i}}\right] P^{\prime}{ }_{k}(\psi) \sqrt{1-\psi^{2}}
\end{aligned}
$$

Further, using strain-displacements equation (4) and stress-strain equation (2), we can find strains and stresses in radial and tangential directions as

$$
\begin{aligned}
& \varepsilon_{r r}=\sum_{i=1}^{2} \rho_{0 i} R_{0 i} r^{\rho_{0 i}-1}+\sum_{k=1}^{\infty}\left[\sum_{i=1}^{4} \rho_{k i} R_{k i} r^{\rho_{k i}-1}\right] P_{k}(\psi) \\
& \varepsilon_{\theta \theta}=\sum_{i=1}^{2} R_{0 i} r^{\rho_{0 i}-1}+\sum_{k=1}^{\infty}\left[\sum_{i=1}^{4} R_{k i} r^{\rho_{k i}-1}\right] P_{k}(\psi)+\sum_{k=1}^{\infty}\left[\sum_{i=1}^{4} D_{k i} R_{k i} r^{\rho_{k i}-1}\right](k(k+ \\
& \text { 1) } \left.P_{k}(\psi)-\psi P^{\prime}{ }_{k}(\psi)\right) \\
& \varepsilon_{\phi \phi}=\sum_{i=1}^{2} R_{0 i} r^{\rho_{0 i}-1}+\sum_{k=1}^{\infty}\left[\sum_{i=1}^{4} R_{k i} r^{\rho_{k i}-1}\right] P_{k}(\psi)+\sum_{k=1}^{\infty}\left[\sum_{i=1}^{4} D_{k i} R_{k i} r^{\rho_{k i}-1}\right] \psi P_{k}^{\prime}(\psi) \\
& \varepsilon_{r \theta}=\sum_{k=1}^{\infty}\left[\sum_{i=1}^{4}\left(D_{k i}\left(\rho_{k i}-1\right)-1\right) R_{k i} r^{\rho_{k i}-1}\right] P^{\prime}{ }_{k}(\psi) \frac{\sqrt{1-\psi^{2}}}{2} \\
& \sigma_{r r}=(\xi+2 \eta)\left(\sum_{i=1}^{2} \rho_{0 i} R_{0 i} r^{\rho_{0 i}-1}+\sum_{k=1}^{\infty}\left[\sum_{i=1}^{4} \rho_{k i} R_{k i} r^{\rho_{k i}-1}\right] P_{k}(\psi)\right)+\xi\left(2 \sum_{i=1}^{2} R_{0 i} r^{\rho_{0 i}-1}+\right. \\
& \left.2 \sum_{k=1}^{\infty}\left[\sum_{i=1}^{4} R_{k i} r^{\rho_{k i}-1}\right] P_{k}(\psi)\right)+\xi\left(\sum_{k=1}^{\infty}\left[\sum_{i=1}^{4} D_{k i} R_{k i} r^{\rho_{k i}-1}\right] k(k+1) P_{k}(\psi)\right) \\
& \sigma_{\theta \theta}=\xi\left(\sum_{i=1}^{2} \rho_{0 i} R_{0 i} r^{\rho_{0 i}-1}+\sum_{k=1}^{\infty}\left[\sum_{i=1}^{4} \rho_{k i} R_{k i} r^{\rho_{k i}-1}\right] P_{k}(\psi)\right)+2(\xi+ \\
& \eta)\left(\sum_{i=1}^{2} R_{0 i} r^{\rho_{0 i}-1}+\sum_{k=1}^{\infty}\left[\sum_{i=1}^{4} R_{k i} r^{\rho_{k i}-1}\right] P_{k}(\psi)\right)+(\xi+2 \eta) \sum_{k=1}^{\infty}\left[\sum_{i=1}^{4} D_{k i} R_{k i} r^{\rho_{k i}-1}\right](k(k+ \\
& \text { 1) } \left.P_{k}(\psi)-\psi P^{\prime}{ }_{k}(\psi)\right)+\xi \sum_{k=1}^{\infty}\left[\sum_{i=1}^{4} D_{k i} R_{k i} r^{\rho_{k i}-1}\right] \psi P^{\prime}{ }_{k}(\psi)
\end{aligned}
$$


$\sigma_{\phi \phi}=\xi\left(\sum_{i=1}^{2} \rho_{0 i} R_{0 i} r^{\rho_{0 i}-1}+\sum_{k=1}^{\infty}\left[\sum_{i=1}^{4} \rho_{k i} R_{k i} r^{\rho_{k i}-1}\right] P_{k}(\psi)\right)+2(\xi+$

$\eta)\left(\sum_{i=1}^{2} R_{0 i} r^{\rho_{0 i}-1}+\sum_{k=1}^{\infty}\left[\sum_{i=1}^{4} R_{k i} r^{\rho_{k i}-1}\right] P_{k}(\psi)\right)+\xi \sum_{k=1}^{\infty}\left[\sum_{i=1}^{4} D_{k i} R_{k i} r^{\rho_{k i}-1}\right] k(k+$

1) $P_{k}(\psi)+2 \eta\left(\sum_{k=1}^{\infty}\left[\sum_{i=1}^{4} D_{k i} R_{k i} r^{\rho_{k i}-1}\right] \psi P^{\prime}{ }_{k}(\psi)\right)$

$\sigma_{r \theta}=\eta\left(\sum_{k=1}^{\infty}\left[\sum_{i=1}^{4}\left(D_{k i}\left(\rho_{k i}-1\right)-1\right) R_{k i} r^{\rho_{k i}-1}\right] P_{k}^{\prime}(\psi) \sqrt{1-\psi^{2}}\right)$

In the equations (19) - (27), four unknowns $R_{k i}(i=1,2,3,4)$ can be evaluated by applying any four boundary conditions picked from the following mechanical boundary conditions as

$$
\begin{aligned}
& u\left(r_{1}, \psi\right)=f_{1}(\psi), u\left(r_{2}, \psi\right)=f_{2}(\psi), w\left(r_{1}, \psi\right)=0, w\left(r_{2}, \psi\right)=0 . \\
& \sigma_{r r}\left(r_{1}, \psi\right)=f_{3}(\psi), \sigma_{r r}\left(r_{2}, \psi\right)=f_{4}(\psi), \sigma_{r \psi}\left(r_{1}, \psi\right)=0, \sigma_{r \psi}\left(r_{2}, \psi\right)=0
\end{aligned}
$$

where $f_{j}(\psi)$ for $j=1,2,3,4$ are some known functions of $\psi$ on inner and outer surfaces of the sphere.

\section{Numerical Results and Discussion}

Assume the inner and outer radii of the sphere, respectively as $r_{1}=1.2, r_{2}=1.4$ in meters; Poisson's ratio $v=0.3$ and constant Young's modulus $Y_{0}=200 \mathrm{GPa}$ for a FGM thick hollow cylinder and mechanical boundary conditions are defined as

$\sigma_{r r}\left(r_{1}, \theta\right)=200 \cos 4 \theta \mathrm{MPa}, \sigma_{r \theta}\left(r_{1}, \theta\right)=0, u\left(r_{2}, \theta\right)=0, w\left(r_{2}, \theta\right)=0$

where radial stress is varying harmonically along tangential direction at inner radius.

Figures 2 and 3 represent radial and circumferential displacement distributions over $r$ and $\theta$ space for the material parameter index $\beta=2$. Here, radial displacement is greater than circumferential displacement in magnitude. Radial displacement $(u)$ and circumferential displacement $(w)$ are zero at external radius and hence they are satisfying harmonic boundary conditions.

Stresses can be seen graphically for the material parameter $\beta=2$ as shown in Figures 4-7. In Figures 2 and 3 radial stresses are decreasing but circumferential stresses are oscillating near zero and 3.5 radian as rleads from inner to outer radius. In Figure 4, peak value of radial stresses is higher at internal radius and lower at external radius in magnitude. In Figures 5 and 6, stresses in $\theta$ and $\phi$ directions are almost equal due to spherically symmetric stress distribution caused by defined harmonic boundary conditions. By looking at Figures 4-7, it can be observed that radial stresses are higher than circumferential and shear stresses in magnitude. 


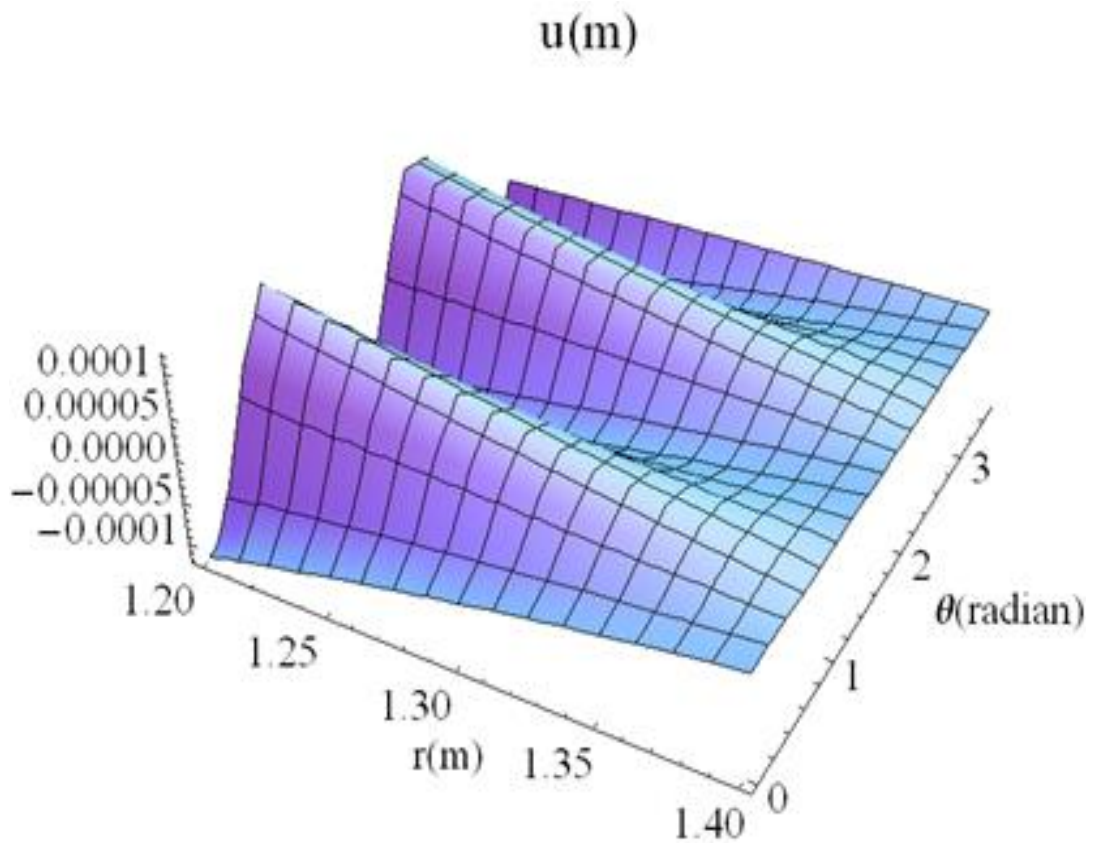

Figure 2. Radial displacement at $\beta=2$.

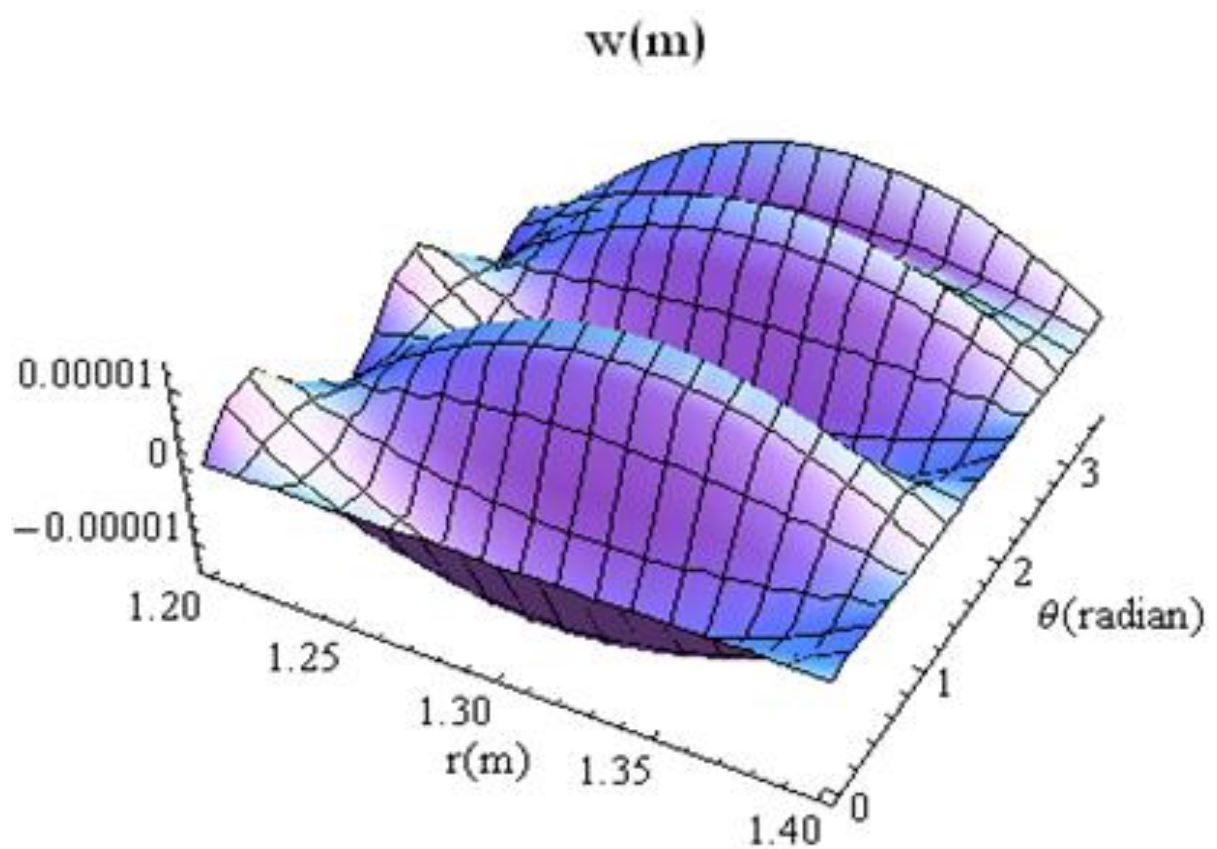

Figure 3. Circumferential displacement at $\beta=2$. 


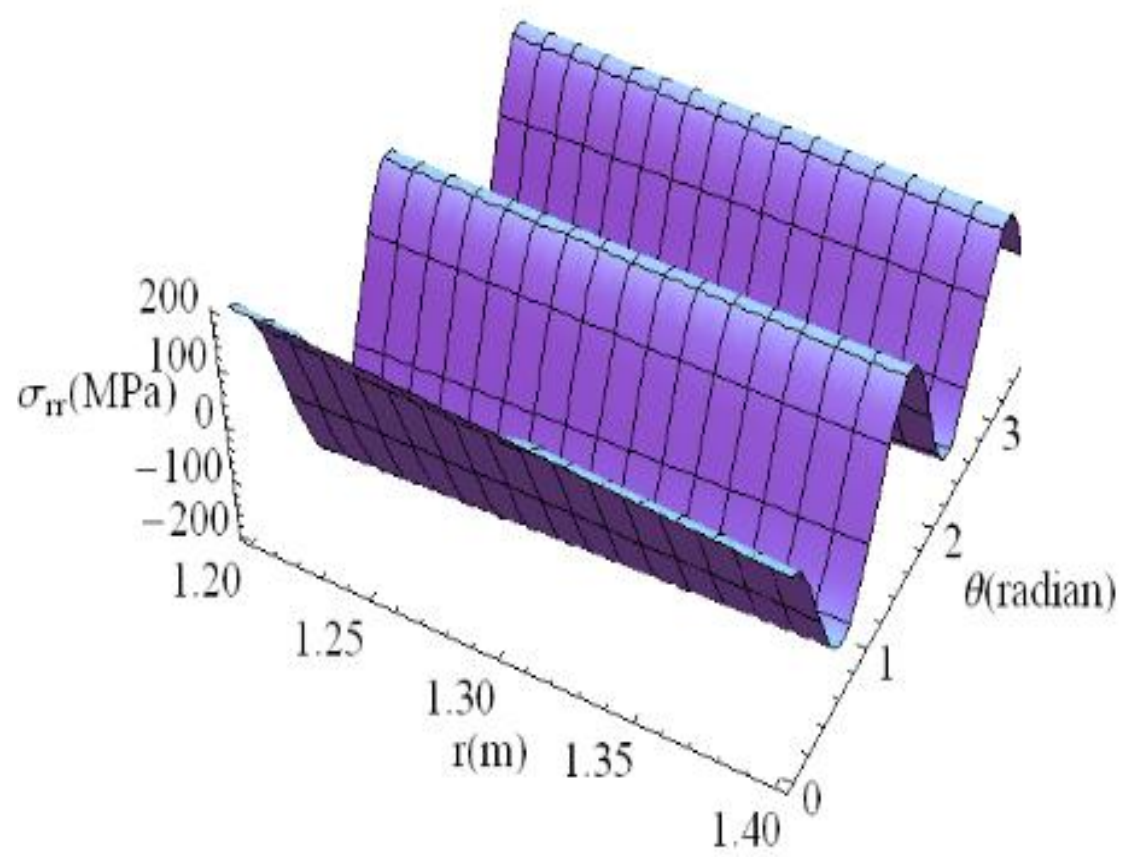

Figure 4. Radial stress at $\beta=2$.

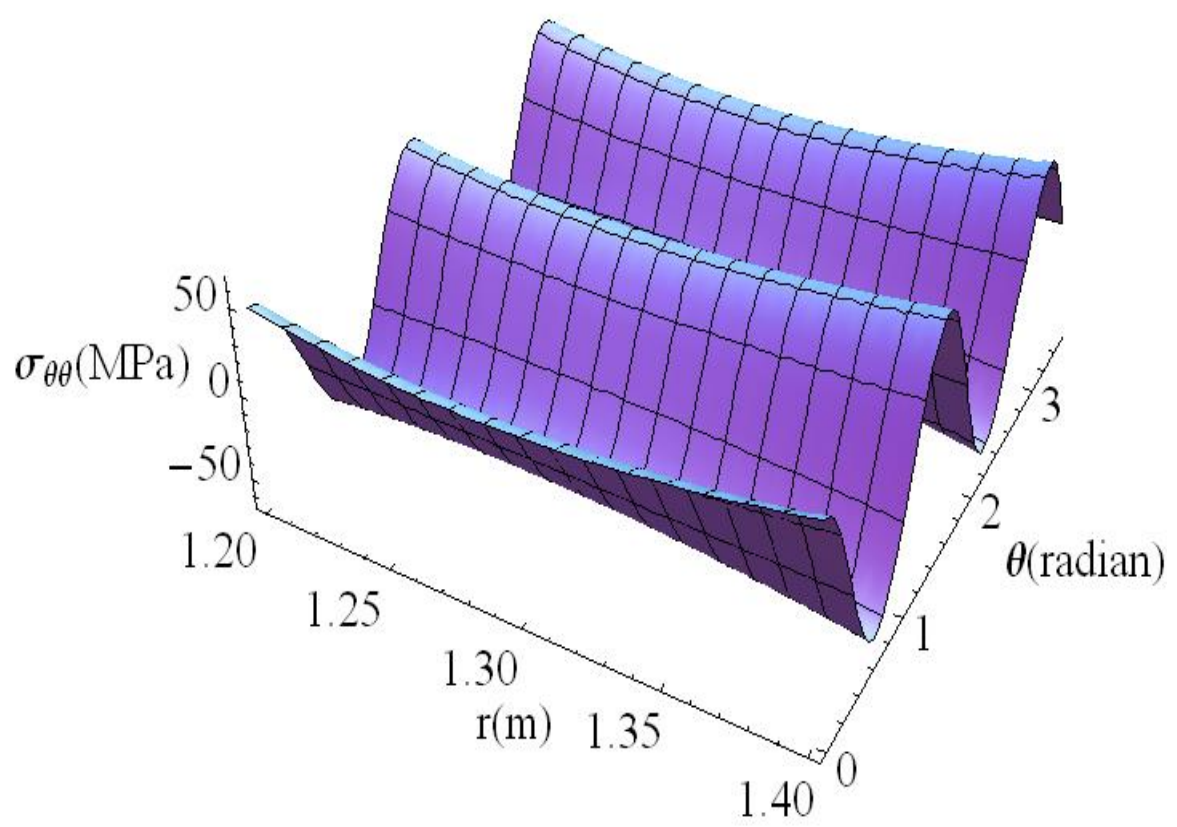

Figure 5. Circumferential stress at $\beta=2$. 


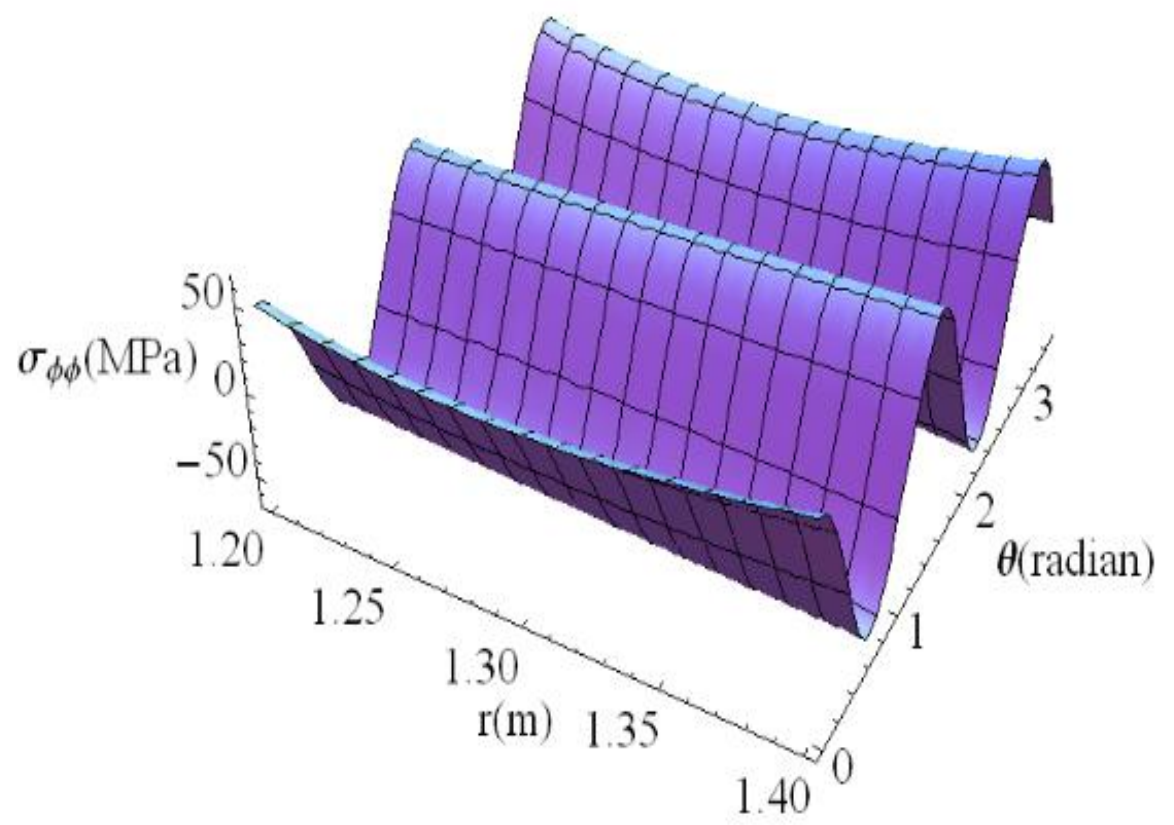

Figure 6. Azimuthal stress at $\beta=2$.

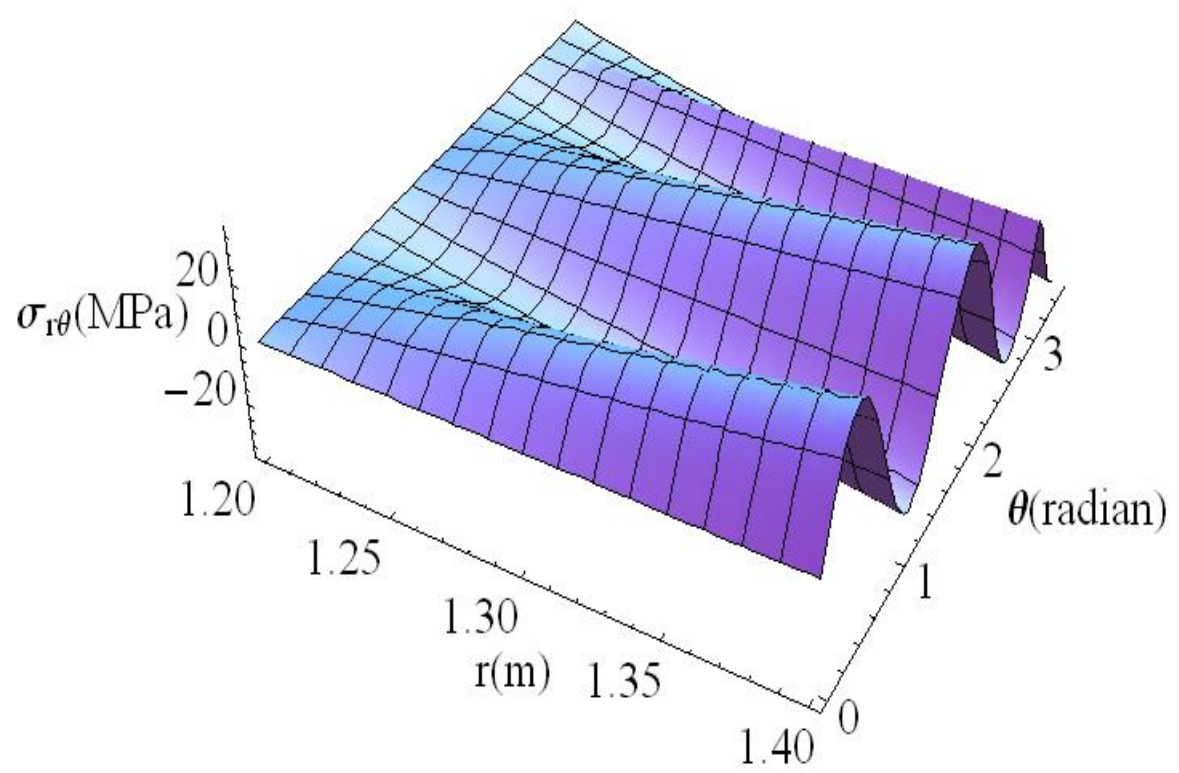

Figure 7. Shear stress at $\beta=2$. 
On the other hand, shear stresses are lower than radial and circumferential stresses in magnitude. In Figure 7, shear stresses are zero at inner radius for all $\theta$ and hence satisfies defined boundary condition. Peak value of shear stress occurs at outer radius along $\theta$. Peak value of magnitudes of shear stresses near 3.5 radian is bigger than the peak value of magnitudes of shear stresses near zero radian as $r$ varies from inner to outer surface.

\section{Conclusions}

This paper presents an analytical solution for mechanical response of functionally graded thickwalled spherical pressure vessel. Nonlinear variation is considered for Young's modulus along thickness direction and Poisson's ratio is taken as constant. In this study, we have considered nonlinear variation of Young's modulus in an exponential form which is a generalization of the power law considered by various authors in the literature. This study can be validated with the homogeneous case in the previous work that is done by Poultangari et al. (2008). They have worked with temperature profile for the problem but we have not considered thermal profile in this work. The author has not considered this generalized form of power law that we have used in this study. Instead of traditional potential function method, Legendre polynomial and Euler differential equations are used to find the stresses. Displacements, strains and stresses are calculated by applying harmonic boundary conditions. An advantage of spherical pressure vessel is that it has smaller surface area per unit volume than any other shape of vessels. Having this property, quantity of pressure can be smoothly distributed throughout the surface to reduce stress concentration in the material. In order to get acceptable results, this series solution may contain a greater number of terms in the case of non-smooth boundary condition and a smaller number of terms in the case of smooth boundary condition. In this work, harmonic boundary conditions are varying on smooth functions so the series solution may converge rapidly by considering a smaller number of terms in the series.

\section{Conflict of Interest}

The authors confirm that there is no conflict of interest to declare for this publication.

\section{Acknowledgements}

This research work is not supported by any funding from public, commercial or not-for-profit sector.

\section{References}

Aragh, B.S., \& Hedayati, H. (2012). Static response and free vibration of two-dimensional functionally graded metal/ceramic open cylindrical shells under various boundary conditions. International Journal of Acta Mechanica, 223(2), 309-330.

Asgari, M., Akhlaghi, M., \& Hosseini, S.M. (2009). Dynamic analysis of two-dimensional functionally graded thick hollow cylinder with finite length under impact loading. International Journal of Acta Mechanica, 208(3), 163-180.

Evci, C., \& Gülgeç, M. (2018). Functionally graded hollow cylinder under pressure and thermal loading: Effect of material parameters on stress and temperature distributions. International Journal of Engineering Science, 123, 92-108.

Gharibi, M., Zamani Nejad, M., \& Hadi, A. (2017). Elastic analysis of functionally graded rotating thick cylindrical pressure vessels with exponentially-varying properties using power series method of Frobenius. Journal of Computational Applied Mechanics, 48(1), 89-98. 
Jabbari, M., Sohrabpour, S., \& Eslami, M.R. (2003). General solution for mechanical and thermal stresses in a functionally graded hollow cylinder due to non-axisymmetric steady-state loads. Journal of Applied Mechanics, 70(1), 111-118.

Kim, K.S., \& Noda, N. (2002). Green's function approach to unsteady thermal stresses in an infinite hollow cylinder of functionally graded material. International Journal of Acta Mechanica, 156(3-4), 145-161.

Loghman, A., Nasr, M., \& Arefi, M. (2017). Non-symmetric thermomechanical analysis of a functionally graded cylinder subjected to mechanical, thermal, and magnetic loads. Journal of Thermal Stresses, 40(6), 765-782.

Mahbadi, H. (2017). Stress intensity factor of radial cracks in isotropic functionally graded solid cylinders. Journal of Engineering Fracture Mechanics, 180, 115-131.

Mehta, P., \& Sahni, M. (2020). Thermo-Mechanical analysis for an axisymmetric functionally graded rotating disc under linear and quadratic thermal loading. International Journal of Mathematical, Engineering and Management Sciences, 5(4),744-757.

Nejad, M.Z., \& Fatehi, P. (2015). Exact elasto-plastic analysis of rotating thick-walled cylindrical pressure vessels made of functionally graded materials. International Journal of Engineering Science, 86, 26-43.

Paul, S.K., \& Sahni, M. (2019). Two-dimensional mechanical stresses for a pressurized cylinder made of functionally graded material. Journal of Structural Integrity and Life, 19(2), 79-85.

Pawar, S.P., Deshmukh, K.C., \& Verma, J. (2017). Thermal behaviour of functionally graded solid sphere with nonuniform heat generation. Journal of Thermal Stresses, 40(1), 86-95.

Poultangari, R., Jabbari, M., \& Eslami, M.R. (2008). Functionally graded hollow spheres under nonaxisymmetric thermo-mechanical loads. International Journal of Pressure Vessels and Piping, 85(5), 295-305.

Reddy, J.N., \& Chin, C.D. (1998). Thermomechanical analysis of functionally graded cylinders and plates. Journal of Thermal Stresses, 21(6), 593-626.

Sahni, M., \& Sharma, S. (2017). Elastic-plastic deformation of a thin rotating solid disk of exponentially varying density. Research on Engineering Structures and Materials, 3(2), 123-133.

Selvamani, R., \& Ponnusamy, P. (2011). Mathematical modelling of waves in a homogeneous isotropic rotating cylindrical panel. Romanian Journal of Mathematics and Computer Science, 1, 12-28.

Seyyed Nosrati, A., Parvizi, A., Afzal, S.A., \& Alimirzaloo, V. (2019). Elasto-plastic solution for thickwalled spherical vessels with an inner FGM layer. Journal of Computational Applied Mechanics, 50(1), $1-13$.

Sharma, P.K., \& Mishra, K.C. (2017). Analysis of thermos-elastic response in functionally graded hollow sphere without load. Journal of Thermal Stresses, 40(2), 185-197.

Singh, T.P., \& Sahni, M. (2016). Study of strength of rotating discs of innovative composite material with variable thickness. In Proceedings of the International Multi-Conference of Engineers and Computer Scientists (Vol. 2). Hong Kong, China.

Wang, B.L., \& Noda, N. (2001). Thermally induced fracture of a smart functionally graded composite structure. Theoretical and Applied Fracture Mechanics, 35(2), 93-109.

Xu, C., Yu, Z.X., \& Du, F. (2019). Dynamic analysis of a thick hollow cylinder made of two-dimensional functionally graded material using time-domain spectral element method. Mechanics of Advanced Materials and Structures, 26(18), 1518-1535. 